\title{
Fat Embolism Syndrome in a Patient who Underwent Unilateral Total Knee Replacement
}

\author{
Vaishali S Kulkarni
}

\begin{abstract}
Fat embolism syndrome (FES) is known to be relatively common in cases of multiple traumatic fractures; it is rare in cases of total knee arthroplasty. We describe a case of a 61-year-old female who underwent unilateral total knee arthroplasty, 5 hours later she developed slurring of speech, disorientation subsequently desaturated, requiring intubation. The clinical diagnosis of fat embolism syndrome was made by criteria of exclusion. Fat embolism syndrome can occur unexpectedly in elective reconstructive orthopedic procedures. One should have a high degree of clinical suspicion of fat embolism syndrome when a patient deteriorates perioperatively. The treatment is primarily supportive.
\end{abstract}

Keywords: Fat embolism syndrome, Local anesthetics, Total knee replacement, Pulmonary embolism.

How to cite this article: Kulkarni VS. Fat Embolism Syndrome in a Patient who Underwent Unilateral Total Knee Replacement. Res Inno in Anesth 2018;3(2):56-59.

Source of support: Nil

Conflict of interest: None

\section{INTRODUCTION}

Fat embolism is caused by the release of fat globules into the systemic circulation and is a known complication after pelvic and long bone fractures; however, it can also present within the setting of elective orthopedic surgery, severe burns, liver injury, closed-chest cardiac massage, and liposuction. ${ }^{1}$ Fat embolism may be asymptomatic but in $1-5 \%$, it results in fat embolism syndrome which ranges from mild respiratory distress to multiorgan dysfunction involving the lung, brain, and skin with a triad of symptoms that include dyspnea, petechial hemorrhages, and neurological abnormality. ${ }^{2}$ Although fat embolism is common following long bone fractures and orthopedic surgery, FES with serious clinical manifestations known to occur in only $0.1 \%$ of patients undergoing joint replacement

Consultant

Department of Anesthesia, Global Hospital, Mumbai, Mumbai, Maharashtra, India

Corresponding Author: Vaishali S Kulkarni, Consultant, Department of Anesthesia, Global Hospital, Mumbai, Mumbai, Maharashtra, India, email: dr_vskulkarni@yahoo.com surgery. ${ }^{3}$ So, we present an interesting case of FES of a patient who developed sudden dyspnea and disorientation after total knee replacement.

\section{CASE REPORT}

A 61-year-old female patient weighing 80 kilograms was diagnosed with osteoarthritis of both knees and admitted for left total knee replacement. She had type 2 diabetes mellitus, hypertension, and hypothyroidism for 3 years. She had an episode of acute angina 1 year earlier for which angioplasty was done, and three coronary stents were placed. The patient was on thyroxine, antihypertensives, oral hypoglycemic agents, tablet clopidogrel and aspirin daily. Tablet clopidogrel was stopped five days before surgery. Preoperative blood and biochemistry tests performed before surgery were within normal ranges. Similarly, no special findings were observed on $\mathrm{X}$-ray chest and electrocardiogram. The preoperative echocardiogram showed good left ventricular function with a left ventricular ejection fraction (LEVF) $-50 \%$ and grade 1 diastolic dysfunction.

The patient received tablets thyroxine $50 \mathrm{mg}$, amlodipine $5 \mathrm{mg}$, nicorandil $10 \mathrm{mg} 2$ hours before surgery. With standard monitoring in the operation theatre and a baseline BP of $160 / 80 \mathrm{~mm} \mathrm{Hg}$, PR of $80 / \mathrm{min}$ and a room air saturation of $99 \%$, she was made to sit, and the subarachnoid block was given under aseptic precautions at the L3 L 4 interspace using a 25 G Quincke's needle. Cerebrospinal fluid (CSF) was clear, and $3.4 \mathrm{~mL}$ of $0.5 \%$ of heavy bupivacaine was given. The intravenous fluid used was Plasma-Lyte and preoperatively iv cefuroxime $1.5 \mathrm{gm}$, iv gentamycin $80 \mathrm{mg}$ and iv tranexamic acid $1.5 \mathrm{gm}$ was given. During surgery, $4 \mathrm{~L} / \mathrm{min}$ oxygen was administered via Hudson faces mask. Vital signs of the patient were stable throughout the entire surgery. Pulse oxygen saturation was maintained at 100. Left side knee replacement was done without using a tourniquet. Operation time was 2 hours and 45 minutes. At the end of surgery, an adductor canal block was given under ultrasound guidance, and aseptic precautions with $20 \mathrm{~mL}$ of $0.125 \%$ bupivacaine and $1 \%$ lignocaine and a catheter were secured in the canal. Postoperatively patient was conscious, oriented, comfortable and moving both lower limbs. 
Five hours later, the patient developed slurring of speech, became drowsy and disoriented and had two episodes of vomiting, and the saturation was at $87 \%$ on room air and oxygen by Hudson mask 4 liters was started after which the saturation of $100 \%$ was attained. The patient was then advised to get a computed tomography (CT) brain and then shifted to intensive care unit (ICU) for further management. During the transfer, the patient developed two episodes of GTCS which was treated with IV midazolam and IV levetiracetam. In the ICU the patient then became restless dyspneic, developed bilateral wheeze. Due to deteriorating GCS and respiratory distress, the patient was intubated and mechanically ventilated. A chest $\mathrm{X}$-ray showed rightsided patch and the arterial blood gas showed a $\mathrm{pH}$ of 7.28, pO2-71, pCO2-32, HCO3-17 and lactate of 5.8. Electrocardiogram and a fresh echocardiogram showed no abnormality. CT brain showed some evidence of pituitary hemorrhage for which the neurologist was consulted and a magnetic resonance imaging (MRI) brain was done which showed no abnormality. She was started on fentanyl and midazolam infusions and insulin infusion as per her blood sugar level and Nitroglycerin infusion in view of elevated blood pressure. She was started on low molecular weight heparin, IV levetiracetam after consulting the neurologist.

Computed tomography (CT) chest showed large patchy collapse consolidation of both lower lobes and mild bilateral pleural effusion and pulmonary angiogram and D-dimer was done which were both normal. There was difficulty to wean the patient off the ventilator due to increased irritability on stopping the sedation and a persistent $\mathrm{V} / \mathrm{Q}$ mismatch, and her respiratory condition further deteriorated on the 3rd postoperative day. She was treated as ARDS with aspiration pneumonitis with $\mathrm{N}$ acetylcysteine and regular nebulization, chest physiotherapy and antibiotics. Urine analysis and fundoscopy was done and showed no fat globules. On the 5th postoperative day, the patient developed a petechial rash over the trunk. The patient developed consumption coagulopathy, the hemoglobin dropped from $10 \mathrm{mg} / \mathrm{dL}$ postoperative to $6.7 \mathrm{mg} / \mathrm{dL}$ and she was transfused two unit of packed red blood cells. Due to patient persistent respiratory distress she was sedated and ventilated for two more days. On the 7th postoperative day she was gradually weaned off the ventilator, and after confirmation of good neurological status, the patient was extubated. The postextubation patient was started on incentive spirometry and active limb physiotherapy and tapered off nasal oxygen and started on oral feeds. The patient was mobilized the next day and shifted to the ward. The patient was discharged on the 11th postoperative day with no neurological sequele.

\section{DISCUSSION}

The pathophysiology of fat embolism syndrome is unclear, but two mechanisms have been suggested. One mechanism suggests that physical obstruction of the systemic vasculature is caused by fat globules released from the bone marrow of long bones due to increased intramedullary pressure after trauma or orthopedic surgery such as placement of intramedullary rods. ${ }^{4}$ The other is a biochemical mechanism that suggests that hydrolyzation of the fat, over a few hours, in plasma to free fatty acids can cause a local inflammatory response and direct toxicity to the lung or capillary endothelium. ${ }^{5}$ Last, the coagulation theory proposed that the release of tissue thromboplastin, following traumatic long bone injury, result in activation of the complement system and extrinsic coagulation pathways and factor VII activation that leads to intravascular coagulation and subsequently lead to an increase in pulmonary permeability of the fat globules. ${ }^{6}$

The initial trigger for FES in TKR surgery was proposed to be due to cementing techniques and increased systemic monomer. On the contrary, the development of FES was described to be due to release of the bone marrow fat globules into circulation before cement application after placement of the intramedullary femoral alignment guide during TKA. The modification of surgical techniques to cementless TKR, and staging (unilateral vs. bilateral) TKR procedures or the use of computer-assisted navigation has not been associated with a lower incidence of FES. ${ }^{3}$ The time onset of the clinical development of FES following knee arthroplasty was variable from an intraoperative cardiorespiratory collapse, before or after deflating the tourniquet, to the development of respiratory, cardiovascular and cerebral dysfunction minutes to hours in the postoperative period.

The presentation of the FES can be subclinical, clinical or fulminating. There is no specific sign, symptom nor test, which is pathognomonic to the FES. The diagnosis of fat embolism is usually a clinical diagnosis of exclusion and requires a vigilant high index of suspicion. Christie et al. documented embolic evidence of fat on echocardiography in 97 out of 110 patients having reaming of a fractured femur or tibia. ${ }^{7}$ Despite the regular occurrence of microscopic fat embolization, FES with multiorgan involvement is rare. The basic triad of FES includes respiratory distress, neurological abnormality, and petechial rash, and other associated features include thrombocytopenia, fever, and unexplained anemia.

For the diagnosis of fat embolism there are no universal criteria; however various criteria have been proposed by different authors. These include Gurd's criteria, Lindeque's criteria, Schonfeld's index. ${ }^{8}$ 


\section{Gurd's Criteria for Diagnosis of Fat Embolism Syndrome ${ }^{9}$}

\section{Major Criteria (One Necessary for Diagnosis)}

- Petechial rash

- Respiratory insufficiency

- Cerebral involvement

\section{Minor Criteria (Four Necessary for Diagnosis)}

- Tachycardia $>120$ beats $/ \mathrm{min}$

- Fever

- Retinal changes: fat or petechiae

- Jaundice

- Renal signs: anuria or oliguria

- Thrombocytopenia

- Anemia

- High erythrocyte sedimentation rate

- Fat macroglobinemia

Criteria developed by Gurd and Wilson in 1974 has been used widely, and at least one of the major criteria and four of the minor criteria are necessary for diagnosing fat embolism syndrome. ${ }^{9}$ Our patient exhibited all of the major symptoms of Gurd's diagnostic criteria, as well as the minor criteria of tachycardia, fever of $38^{\circ} \mathrm{C}$ and anemia. There was no evidence of fat globules in our patient's urine and fundoscopy however the recovery of fat globules has been found to be of uncertain significance as studies have demonstrated fat globules in $>50 \%$ of patients with fractures but no symptoms suggestive of FES. In $20-50 \%$ of patients, the petechial rash is known to appear within 24-36 hours and resolves spontaneously within one week..$^{10}$ Our patient also developed a petechial rash over her trunk on the 3rd day which resolved within the week. ${ }^{6}$

The $A B G$ revealed a low partial pressure of oxygen and a low partial pressure of $\mathrm{CO}_{2}$ with respiratory alkalosis which was strongly suggestive of ARDS and FES. ${ }^{5}$ The classical chest X-ray of fat embolism syndrome shows multiple flocculent shadows ("snow storm appearance" $),{ }_{1}^{11}$ however, a minority have diffuse or patchy consolidation which is more prominent in the periphery and bases. Focal areas of ground glass opacification with interlobular septal thickening are generally seen on chest $\mathrm{CT} .{ }^{12}$ The chest ray and CT scan of our patient showed patchy consolidation of both lungs with mild ground-glass densities and interlobar septal thickening both suggestive of FES. Lungs scan may show ventilationperfusion mismatch, ${ }^{13}$ this was however not done in our patient.

Since our patient had a history of ischemic heart disease, myocardial infarction or cardiac shock were considered as causes. However, since surgery was performed under regional anesthesia, and blood pressure, pulse, electrocardiogram, oxygen saturation level, were within normal ranges throughout the entire anesthesia period, with no postoperative ECG changes and negative cardiac enzymes, these causes could be ruled out.

The treatment of fat embolism syndrome is primarily supportive of early resuscitation and minimizing the stress response and hypovolemia. The goal should be on maintaining adequate tissue oxygenation and arterial oxygen saturation of more than $90 \%$. The patient's lung disease might necessitate the use of positive airway pressure or even mechanical ventilation. The use of prophylactic steroid therapy has been advocated, but with limited results. Apart from this general supportive measures, including hemodynamic stabilization, maintenance of normal electrolyte values are essential. The prognosis for patients who survive fat embolism is good, with recovery from the fat embolism syndrome usually being complete within 2-4 weeks, although some neurological signs may remain for up to 3 months. ${ }^{14}$

\section{CONCLUSION}

The FES is an unpredictable condition with a varied presentation. There are no obvious determining factors. Thus it is up to the clinician to be highly vigilant with regards to trauma and orthopedic patients. The first step is to be able to recognize the FES and then respond quickly and aggressively in the treatment.

\section{REFERENCES}

1. Taviloglu K, Yanar H. Fat embolism syndrome. Surg Today 2007;37(1):5-8.

2. Yeo SH, Chang HW, Sohn SI, et al. Pulmonary and Cerebral Fat Embolism Syndrome After Total Knee Replacement. J Clin Med Res 2013;5(3):239-242.

3. Hofmann S, Huemer G, Salzer M. Pathophysiology and management of the fat embolism syndrome. Anaesthesia 1998;53(S2):35-37.

4. Hulman G. The pathogenesis of fat embolism. J Pathol 1995;176(1):3-9.

5. Saigal R, Mittal M, Kansal A, et al. Assoc Physicians India 2008;56:245-249.

6. Watson AJ. Genesis of fat emboli. J Clin Pathol (R Coll Pathol) 1970;4(Supp 1):132-142.

7. Christie J, Robinson CM, Pell AC, et al. Transcardiac echocardiography during invasive intramedullary procedures. Bone Jt J 1995;77-B(3):450-455.

8. Schonfeld SA, Ploysongsang Y, DiLisio R, et al. Fat embolism prophylaxis with corticosteroids. A prospective study in highrisk patients. Ann Intern Med 1983;99(4):438-443.

9. Gurd AR, Wilson RI. The fat embolism syndrome. J Bone Joint Surg Br 1974;56B(3):408-416.

10. Kaplan RP, Grant JN, Kaufman AJ. Dermatologic features of the fat embolism syndrome. Cutis 1986;38(1):52-55.

11. Liljedahl SO, Westermark L. Aetiology and treatment of fat embolism. Report of five cases. Acta Anaesthesiol Scand 1967;11(3):177-194. 
12. Van den Brande FGJ, Hellemans S, De Schepper A, et al. Post-traumatic severe fat embolism syndrome with uncommon CT findings. Anaesth Intensive Care 2006;34(1):102-106.
13. Prys-Roberts C, Greenbaum R, Nunn JF, et al. Disturbances of pulmonary function in patients with fat embolism. J Clin Pathol Suppl (R Coll Pathol) 1970;4:143-149.

14. O'Higgins JW. Fat embolism. Br J Anaesth 1970;42(2):163-168. 